\title{
Free Axial Vibrations of Non-Uniform Rods
}

\author{
Shuqi Guo ${ }^{1, a}$,Shaopu Yang ${ }^{2, b}$
}

${ }^{1}$ Department of Engineering Mechanics, Shijiazhuang Tiedao University, Shijiazhuang, 050043, China

${ }^{2}$ School of Mechanical Engineering, Shijiazhuang Tiedao University, Shijiazhuang, 050043, China

aemail: shuqig@yahoo.com.cn, bemail:yangsq@stdu.edu.cn

Keywords: axial vibration, non-uniform rod, series solution, WKB method

\begin{abstract}
Free axial vibrations of non-uniform rods are investigated by a proposed method, which results in a series solution. In a special case, with the proposed method an exact solution with a concise form can be obtained, which imply four types of profiles with variation in geometry or material properties. However, the WKB (Wentzel-Kramers-Brillouin) method leads to a series solution, which is a Taylor expansion of the results of the pro-posed method. For the arbitrary non-uniform rods, the comparison indicates that the WKB method is simpler, but the convergent speed of the series solution resulting from the proposed method is faster than that of the WKB method, which is also validated numerically using an exact solution of a kind of non-uniform rods with Kummer functions.
\end{abstract}

\section{Introduction}

The vibration of non-uniform rods is a subject of considerable scientific and practical interest that has been studied extensively, and is still receiving attention in literature. No attempt will be made here to present a bibliographical account of previous work in this area. A study of vibration of tapered rods indicates that the natural frequencies are only affected slightly by the taper [1]. It was shown that the equation of motion of rods with conical cross sections could be reduced to the form of a wave equation by a change of variable [2]. In a study using an approach Abrate [3] sought exact solutions for the problem. He obtained a closed form solution for rods whose cross-sectional area varies as $A(x)=A_{0}(1+a x / L)^{2}$. Exact analytical solutions also exist for exponential and catenoidal rods [4]. Using appropriate transformations Kumar and Sujith [5] solved the problem with cross-sectional area $A(x)=(a+b x)^{n}$ and $A(x)=A_{0} \sin ^{2}(a+b x)$. In a recent study Anil and Sujith (2005) solved the problem with cross-sectional area $A(x)=k x^{n} e^{b x^{2}}$ and $A(x)=k x^{n} e^{b x}$. Guo and Yang (2010) also investigated the problem with cross-sectional area $A(x)=A_{0} e^{a x+b x^{2}}$ with Kummar functions. Recently, the wave motions in non-uniform one-dimensional waveguides (Guo and Yang, 2011) are studied.

In this paper, a method is proposed to solve the free axial vibration of non-uniform rods, which results in a series solution. And the proposed method is compared with the WKB method. In Section 2 , the governing equations are presented. Section 3 investiges a special case of non-uniform rods. The proposed method is presented in Section 4 to solve the problem. In section 5, the same problem is also investigated by the known WKB method. And the results of the WKB method are compared with those of the proposed method in Section 4. In section 6, in order to examine the convergent speed of the two methods numerically, an exact solution of a kind of non-uniform rods is obtained with Kummer functions. Conclusions are drawn in Section 7.

\section{The governing equations of free vibrations of non-uniform rods}

The concerned non-uniform rod is shown as Fig. 1, in which Young's modulus $E(x)$, density $\rho(x)$ and cross-sectional area $A(x)$ vary with the axial coordinate $x$. Its general governing equation of free axial vibrations reads 


$$
\frac{\partial}{\partial x}\left[E(x) A(x) \frac{\partial u}{\partial x}\right]=\rho(x) A(x) \frac{\partial^{2} u}{\partial t^{2}} \text {. }
$$

In order to simplify the governing equation, we introduce a change of variable (Zhong Weifang and Nie Guohua, 1997), i.e. Eq. (2),

$$
y=\int_{0}^{x} \sqrt{\frac{\rho(\tau) / \rho_{0}}{E(\tau) / E_{0}}} d \tau
$$

where $\rho_{0}$ and $E_{0}$ are the density and Young's modulus at certain section of the rod. Substitution of Eq. (2) into Eq. (1) yields

$$
\frac{\partial}{\partial y}\left[E_{0} \bar{A}(y) \frac{\partial u(y, t)}{\partial y}\right]=\rho_{0} \bar{A}(y) \frac{\partial^{2} u}{\partial t^{2}}
$$

where $\bar{A}(y)=\sqrt{\frac{\rho(x) E(x)}{\rho_{0} E_{0}}} A(x)$. For constant density $\rho(x)=\rho_{0}$ and constant Young's modulus $E(x)=E_{0}$, the governing equations (1) and (3) are reduced to

$$
\frac{\partial}{\partial x}\left[E_{0} A(x) \frac{\partial u(x, t)}{\partial x}\right]=\rho_{0} A(x) \frac{\partial^{2} u}{\partial t^{2}},
$$

which is the governing equation of free axial vibration of non-uniform rods with variable cross sections. It is easy to find that Eqs. (3) and (4) have the same form in mathematics. Then let us focus on the governing equation (4) for simplicity.

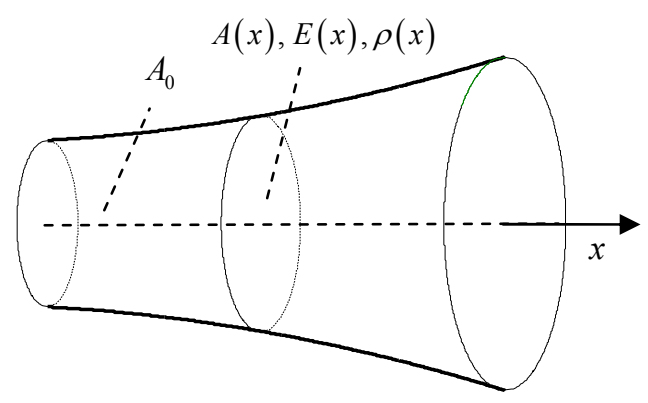

Fig. 1. Section of a non-uniform waveguide.

\section{The free vibrations of a kind of non-uniform rods}

Without loss of generality, let $u(x, t)=U(x) e^{i \omega t}$, substitution of it into Eq. (4) yields

$$
\frac{d^{2} U}{d x^{2}}+\frac{A^{\prime}(x)}{A(x)} \frac{d U}{d x}+\frac{\omega^{2}}{c_{0}^{2}} U=0,
$$

where $c_{0}=\sqrt{E_{0} / \rho_{0}}, U(x)$ is the mode function, the prime denotes differentiation with respect to the argument. In this, a transformation $V(x)=\sqrt{A(x)} U(x)$ is introduced, and Eq. (5) is transformed into Eq. (6), which is the standard form of a second-order ordinary differential equation with variable coefficients.

$$
\frac{d^{2} V(x)}{d x^{2}}+\left(\frac{\omega^{2}}{c_{0}^{2}}-q(x)\right) V(x)=0,
$$

where $q(x)=\frac{d^{2} \sqrt{A(x)}}{d x^{2}} \frac{1}{\sqrt{A(x)}}$. If the function is a real constant, i.e. $q(x)=b$, one could find four profiles with variable cross sections, which are shown as Eq. (7). 


$$
A(x)=\left\{\begin{array}{l}
A_{0}(1+c x)^{2}, \\
A_{0} e^{c x} \\
\frac{A_{0} \cosh ^{2}(c x+s)}{\cosh ^{2} s}, \\
\frac{A_{0} \cos ^{2}(c x+s)}{\cos ^{2} s} .
\end{array}\right.
$$

where $c, s$ and $A_{0}$ are real constants. Then one could find that the studies of Abrate(1995), Eisenberger (1991), Bapat (1995), Kumar and Sujith (1997) are the special case of the results in this section. The solution of Eq. (4) could be written as

$$
u(x, t)=U(x)\left(c_{1} \sin \omega t+c_{2} \cos \omega t\right),
$$

where $c_{1}$ and $c_{2}$ are real constants determined by initial conditions; $U(x)$ is the mode function shown as

$$
U(x)=\sqrt{A_{0} / A(x)}\left[B_{1} \sin \left(x \sqrt{\omega^{2} / c_{0}^{2}-b}\right)+B_{2} \cos \left(x \sqrt{\omega^{2} / c_{0}^{2}-b}\right)\right],
$$

where $B_{1}$ and $B_{2}$ are determined by boundary conditions. The concerned three boundary conditions here are two fixed ends, one fixed end and one free end, and two free ends, which are shown as Eq. (10), respectively.

$$
\begin{aligned}
& U(0)=U(L)=0, \\
& U(0)=U^{\prime}(L)=0, \\
& U^{\prime}(0)=U^{\prime}(L)=0 .
\end{aligned}
$$

Using the mode function Eq. (9) and the boundary conditions Eq. (10), one could find the mode frequency equations and the values of $B_{1}$ and $B_{2}$. For the boundary condition of two fixed ends, $B_{1}=1, B_{2}=0$. And its mode frequency equation reads

$$
k_{j} L=j \pi, j=1,2,3, \ldots,
$$

where $k_{j}=\sqrt{\omega_{j}^{2} / c_{0}^{2}-b}$. For the boundary condition of one fixed end and one free end, $B_{1}=1, B_{2}=0$. Its mode frequency equation reads

$$
2 k_{j} \frac{A(L)}{A^{\prime}(L)}=\tan k_{j} L, j=1,2,3 \ldots
$$

For the boundary condition of two free ends, $B_{1}=1, B_{2}=\frac{2 A_{0} k_{j}}{A^{\prime}(0)}$. Its mode frequency equation reads

$$
\left[4 k_{j}^{2} A_{0} A(L)+A^{\prime}(0) A^{\prime}(L)\right] \sin k_{j} L+2 k_{j}\left[A_{0} A^{\prime}(L)-A(L) A^{\prime}(0)\right] \cos k_{j} L=0, j=1,2,3 \ldots
$$

The proposed method for arbitrary non-uniform rods

Substitution of $\xi=x / L$ into Eq. (6) yields

$$
\frac{d^{2} V(\xi)}{d \xi^{2}}+\left(\Omega^{2}-\theta(\xi)\right) V(\xi)=0,
$$

where $\Omega=\frac{\omega L}{c_{0}}, \theta(\xi)=q(\xi) L^{2}$. For the case of $\Omega^{2}-\theta(\xi)<0$, axial waves could not propagate along the rod (Guo and Yang, 2011). Here we consider the case of $\Omega^{2}-\theta(\xi)>0$. Assume the solution of Eq. (12) is the form of

$$
V(\xi)=\Lambda(\xi) \mathrm{e}^{\mathrm{i} h(\xi)},
$$

where $\Lambda(\xi)$ and $h(\xi)$ are undetermined real functions, $\mathrm{i}=\sqrt{-1}$. With the help of $V(x)=\sqrt{A(x)} U(x)$ and Eq. (13), the mode function could be written as

$$
U(\xi)=a(\xi)\left(B_{1} \sin h(\xi)+B_{2} \cos h(\xi)\right),
$$

where $a(\xi)=\Lambda(\xi) / \sqrt{A(\xi)}$ is the amplitude function, whose solution is shown as Eq. (18). 
Substitution of Eq. (13) into Eq. (12) yields Eqs. (15) and (16).

$$
\begin{aligned}
& 2 H \Lambda^{\prime}+\Lambda H^{\prime}=0, \\
& \Lambda^{\prime \prime}-H^{2} \Lambda+\left(\Omega^{2}-\theta(\xi)\right) \Lambda=0,
\end{aligned}
$$

where $H(\xi)=\frac{d h(\xi)}{d \xi}$. The solution of Eq. (15) could be obtained as

$$
\Lambda(\xi)=\Lambda\left(\xi_{0}\right) \sqrt{\frac{H\left(\xi_{0}\right)}{H(\xi)}} .
$$

Substituting it into $a(\xi)=\Lambda(\xi) / \sqrt{A(\xi)}$ yields

$$
a(\xi)=a\left(\xi_{0}\right) \sqrt{\frac{H\left(\xi_{0}\right) A\left(\xi_{0}\right)}{H(\xi) A(\xi)}},
$$

where $a\left(\xi_{0}\right), H\left(\xi_{0}\right)$ and $A\left(\xi_{0}\right)$ are the values of $a(\xi), H(\xi)$ and $A(\xi)$ at certain point $\xi=\xi_{0}$. Substitution of Eq. (17) into Eq. (16) yields

$$
\frac{3}{4} \frac{\left(\frac{d H(\xi)}{d \xi}\right)^{2}}{H^{2}(\xi)}-\frac{1}{2} \frac{\frac{d^{2} H(\xi)}{d \xi^{2}}}{H(\xi)}-H^{2}(\xi)+\Omega^{2}-\theta(\xi)=0 .
$$

If the first two terms of eq. (19) could be neglected, one could find an approximate solution, i.e. $H^{2}(\xi)=\Omega^{2}-\theta(\xi)$.

Substitution of Eq. (20) into Eq. (19), and let $\beta(\xi)=\Omega^{2}-\theta(\xi)$, one could find that the left hand of Eq. (19) is

$$
\frac{5}{16 \beta^{2}}\left(\frac{d \theta(\xi)}{d \xi}\right)^{2}+\frac{1}{4 \beta(\xi)} \frac{d^{2} \theta(\xi)}{d \xi^{2}}=\mathrm{O}\left(\frac{1}{\beta(\xi)}\right),
$$

whose order is the same with $\frac{1}{\beta(\xi)}$. Then we could assume that a series solution of Eq. (19) is the form of

$$
H(\xi)=\sqrt{\beta(\xi)+\sum_{n=1}^{\infty} \frac{b_{n}(\xi)}{\beta^{n}(\xi)}} .
$$

In order to determine the expressions of coefficients $b_{n}(\xi)$, substituting it into Eq. (19) and doing some arithmetic work, one could obtain Eq. (22). Eq. (22) could be further transformed into Eq. (23) after collecting like terms with the same power of $\beta$.

$$
\begin{aligned}
& \left(\theta^{\prime \prime}-\sum_{n=1}^{\infty}\left(\frac{n b_{n} \theta^{\prime \prime}}{\beta^{n+1}}+\frac{\left(n^{2}+n\right) b_{n} \theta^{\prime 2}}{\beta^{n+2}}+\frac{4 b_{n}}{\beta^{n-1}}+\frac{b_{n, \xi \xi}}{\beta^{n}}+\frac{2 n \theta^{\prime} b_{n, \xi}}{\beta^{n+1}}\right)-4\left(\sum_{n=1}^{\infty} \frac{b_{n}}{\beta^{n}}\right)^{2}\right) H^{2}(\xi) \\
& +\frac{5}{4}\left(\theta^{\prime}-\sum_{n=1}^{\infty} \frac{n b_{n} \theta^{\prime}}{\beta^{n+1}}-\sum_{n=1}^{\infty} \frac{b_{n, \xi}}{\beta^{n}}\right)^{2}=0 \\
& \left(\theta^{\prime \prime}-4 b_{1}\right) \beta-4 b_{2}-b_{1, \xi \xi}+\frac{5}{4} \theta^{\prime 2}-\sum_{n=1}^{\infty} \frac{4 b_{n+2}+\bar{b}_{n}}{\beta^{n}}+\sum_{n=2}^{\infty} \frac{5 \hat{b}_{n} / 4-\tilde{b}_{n}}{\beta^{n}}-4 \sum_{n=i+j+k=3}^{\infty} \frac{b_{i} b_{j} b_{k}}{\beta^{n}}=0,
\end{aligned}
$$

where the comma and prime all denote the differentiation with respect to $\xi$, and

$$
\begin{aligned}
& \bar{b}_{n}=(n+1) b_{n} \theta^{\prime \prime}+(n+2.5)(n-1) b_{n-1} \theta^{\prime 2}+b_{n+1, \xi \xi}+(2 n+2.5) \theta^{\prime} b_{n, \xi}, \\
& \hat{b}_{n}=\sum_{j=1}^{n-1}\left((j-1)(n-j-1) b_{j-1} b_{n-j-1} \theta^{\prime 2}+b_{j, \xi} b_{n-j, \xi}+2(j-1) b_{j-1} b_{n-j, \xi} \theta^{\prime}\right), \\
& \tilde{b}_{n}=\sum_{j=1}^{n-1} b_{n-j}\left[(j-1) b_{j-1} \theta^{\prime \prime}+(j-2)(j-1) b_{j-2} \theta^{\prime 2}+8 b_{j+1}+b_{j, \xi \xi}+2(i-1) \theta^{\prime} b_{j-1, \xi}\right] .
\end{aligned}
$$


The vanishing of coefficients of each order of $\beta^{n}$ in Eq. (23) results in the expressions of $b_{n}(\xi)$. The first four terms are

$$
\begin{aligned}
& b_{1}=\frac{\theta^{\prime \prime}}{4}, b_{2}=-\frac{1}{4} b_{1, \xi \xi}+\frac{5}{16} \theta^{\prime 2}, b_{3}=-2 b_{1}^{2}-\frac{1}{4} b_{2, \xi \xi}-\frac{9}{8} b_{1, \xi} \theta^{\prime}, \\
& b_{4}=\frac{5}{16} b_{1, \xi} b_{1, \xi}-5 b_{1} b_{2}-\frac{1}{4} b_{1} b_{1, \xi \xi}-\frac{9}{8} b_{1} \theta^{\prime 2}-\frac{1}{4} b_{3, \xi \xi}-\frac{13}{8} b_{2, \xi} \theta^{\prime}
\end{aligned}
$$

For $n \geq 5$, the recursive expressions of $b_{n}(\xi)$ could be obtained as

$$
b_{n}=-\frac{1}{4} \bar{b}_{n-2}+\frac{5}{16} \hat{b}_{n-2}-\frac{1}{4} \tilde{b}_{n-2}-\sum_{m=2}^{n-3}\left(\sum_{i=1}^{m-1} b_{i} b_{m-i}\right) b_{n-2-m} \text {. }
$$

Substituting Eq. (24) into Eq. (21), one could find the explicit expression of $H(\xi)$. Integrating it from $\xi_{0}$ to $\xi$ one can obain $h(\xi)$ shown as Eq. (25).

$$
h(\xi)=\int_{\xi_{0}}^{\xi} \sqrt{\Omega^{2}-\theta(s)+\sum_{n=1}^{\infty} \frac{b_{n}(s)}{\left(\Omega^{2}-\theta(s)\right)^{n}}} d s,
$$

where $\xi_{0}$ is an arbitrary real constant. Without loss of generality, let $\xi_{0}=0$ and substituting $h(\xi)=\int_{0}^{\xi} H(s) d s$ into Eq. (14) leads to

$$
U(\xi)=a(\xi)\left(B_{1} \sin \int_{0}^{\xi} H(s) d s+B_{2} \cos \int_{0}^{\xi} H(s) d s\right) .
$$

The corresponding boundary conditions eq. (11) is transformed as

$$
\begin{aligned}
& U(0)=U(1)=0, \\
& U(0)=U^{\prime}(1)=0, \\
& U^{\prime}(0)=U^{\prime}(1)=0 .
\end{aligned}
$$

For the rods with two fixed ends, substitution of Eq. (27a) into Eq. (26) yields $B_{1}=1, B_{2}=0$ and its mode frequency equation Eq. (28):

$$
h(1)=j \pi, j=1,2,3, \ldots
$$

For high order mode frequency, due to $h(1) \approx \int_{0}^{1}\left(\Omega_{n}-\frac{1}{2} \theta(s)\right) d s=n \pi$, one could find an approximate solution

$$
\Omega_{j} \approx j \pi+\frac{1}{2} \int_{0}^{1} \theta(s) d s, \quad j \gg 1 .
$$

For the rods with one fixed end and one free end, substitution of Eq. (27b) into Eq. (26) yields $B_{1}=1, B_{2}=0$ and its mode frequency equation:

$$
a^{\prime}(1) \cos h(1)-\sin h(1) H(1) a(1)=0 \text {. }
$$

For the rods with two free ends, substitution of the boundary condition Eq. (27c) into Eq. (23) yields $B_{1}=1, B_{2}=-H(0) a(0) / a^{\prime}(0)$ and its mode frequency equation is

$$
H(0) \frac{a^{\prime}(1)}{a(1)}-H(1) \frac{a^{\prime}(0)}{a(0)}=\left(H(0) H(1)+\frac{a^{\prime}(0) a^{\prime}(1)}{a(0) a(1)}\right) \tan h(1) .
$$

\section{The WKB method and the comparison with the proposed method}

With the method of WKB (R. E. Langer, 1934), the solution of Eq. (15) could be assumed as the form of

$$
V(\xi)=\exp \left(\mathrm{i} \Omega \int_{\xi_{0}}^{\xi} w(s) d s\right),
$$

where $\mathrm{i}=\sqrt{-1}, \xi_{0}$ is an arbitrary real constant, $w(\xi)$ is an undetermined function. Substitution of Eq. (32) into Eq. (15) yields

$$
\text { i } \Omega \frac{d w(\xi)}{d \xi}-\Omega^{2} w^{2}(\xi)+\Omega^{2}-\theta(\xi)=0 .
$$


Assume the undetermined function $w(\xi)$ could be expanded as

$$
w(\xi)=1+\sum_{n=1}^{\infty} w_{n}(\xi) \Omega^{-n} \text {. }
$$

Substituting it into Eq. (33) and collecting together terms that involve the same powers of $\Omega$, one could find

$$
-2 w_{1} \Omega+\left(\mathrm{i} w_{1, \xi}-2 w_{2}-\theta\right)+\left(\mathrm{i} w_{2, \xi}-2 w_{3}\right) \frac{1}{\Omega}+\sum_{n=2}^{\infty}\left(\mathrm{i} w_{n+1, \xi}-2 w_{n+2}-\sum_{j=1}^{n-1} w_{j+1} w_{n-j+1}\right) \frac{1}{\Omega^{n}}=0 .
$$

In eq. (35) the vanishing of the coefficients of $\Omega^{n}$ results in the expressions of $g_{n}(\xi)$,

$$
\begin{aligned}
& w_{1}=0, w_{2}=-\frac{1}{2} \theta(\xi), w_{3}=-\frac{\mathrm{i}}{4} \theta^{\prime}, w_{4}=\frac{1}{8} \theta^{\prime \prime}-\frac{1}{8} \theta^{2}, w_{5}=\mathrm{i}\left(\frac{1}{16} \theta^{\prime \prime}-\frac{1}{4} \theta \theta^{\prime}\right), \\
& w_{6}=-\frac{1}{32} \theta^{\prime \prime \prime}+\frac{1}{8} \theta \theta^{\prime}+\frac{3}{16} \theta \theta^{\prime \prime}-\frac{1}{16} \theta^{3}+\frac{5}{32}\left(\theta^{\prime}\right)^{2} .
\end{aligned}
$$

For $n \geq 4$,

$$
w_{n}=\mathrm{i} \frac{1}{2} w_{n-1, \xi}-\frac{1}{2} \sum_{j=1}^{n-3} w_{n-j-1} w_{j+1} \text {. }
$$

Using the idea of the proposed method in the previous section, complex $w_{n}(\xi)$ could be further rewritten as

$$
w(\xi)=P(\xi)+\mathrm{i} Q(\xi)=1+\sum_{j=1}^{\infty} p_{j}(\xi) \Omega^{-2 j}+\mathrm{i} \sum_{j=1}^{\infty} q_{j}(\xi) \Omega^{-2 j-1},
$$

where

$$
P(\xi)=1+\sum_{j=1}^{\infty} p_{j}(\xi) \Omega^{-2 j}, Q(\xi)=\sum_{j=1}^{\infty} q_{j}(\xi) \Omega^{-2 j-1} .
$$

Substitution of Eq. (37) into Eq. (33) leads to

$$
\left\{\begin{array}{l}
p_{n+1}=-\frac{1}{2} q_{n, x}-\frac{1}{2} \sum_{s=1}^{n-1}\left(p_{s} p_{n+1-s}-q_{s} q_{n-s}\right), \\
q_{n}=\frac{1}{2} p_{n, x}-\sum_{s=1}^{n-1} p_{s} q_{n-s} .
\end{array}\right.
$$

For $\theta(\xi)=c$, the WKB method in this section results in a series solution of Eq. (12) shown as Eqs. (40) and (41):

$$
\begin{aligned}
& w(\xi)=1-\frac{1}{2} \frac{c}{\Omega^{2}}-\frac{1}{8} \frac{c^{2}}{\Omega^{4}}-\frac{1}{16} \frac{c^{3}}{\Omega^{6}}-\frac{5}{128} \frac{c^{4}}{\Omega^{8}}+\ldots . \\
& V(\xi)=\exp \left(\mathrm{i} \Omega \int_{0}^{\xi} w(s) d s\right)=\exp \left(\mathrm{i} \Omega-\frac{\mathrm{i}}{2} \frac{c}{\Omega}-\frac{\mathrm{i}}{8} \frac{c^{2}}{\Omega^{3}}-\frac{\mathrm{i}}{16} \frac{c^{3}}{\Omega^{5}}-\frac{5 \mathrm{i}}{128} \frac{c^{4}}{\Omega^{7}}+\ldots .\right) .
\end{aligned}
$$

In this case, it is easy to know

$$
H(\xi)=\Omega-\frac{1}{2} \frac{c}{\Omega}-\frac{1}{8} \frac{c^{2}}{\Omega^{3}}-\frac{1}{16} \frac{c^{3}}{\Omega^{5}}-\frac{5}{128} \frac{c^{4}}{\Omega^{7}}+\ldots .
$$

The series Eq. (21) in Section 4 results in an exact solution with a concise form, i.e.

$$
H(\xi)=\sqrt{\Omega^{2}-c} \text {. }
$$

If $\frac{|c|}{\Omega^{2}} \leq 1$, The Taylor expansion of the right side of Eq. (43) is exactly the right side of Eq. (42). It is not a surprise, because the series $P(\xi)=\left(1+\sum_{j=1}^{\infty} p_{j}(\xi) \Omega^{-2 j}\right)$ is the Taylor expansion of $\frac{H(\xi)}{\Omega}$ at $\Omega=\infty$, which is one of the results in Section 4. So, we can expect that the convergent speed of the proposed series is faster than the WKB method. 


\section{Numerical comparison of the two methods}

In order to compare the two methods numerically, we first solve the problem with a special kind of non-uniform rod with variable cross-sectional area $A(x)=A_{0} \exp \left(\frac{c x^{2}}{L^{2}}\right)$. In this case one could obtain its exact solution of free axial vibrations with Kummer function, which is a special case of (Guo, 2010; Ray, 1997). Using a transformation $x=L \sqrt{-\frac{z}{c}}$ and substituting it into Eq. (5) yield Eq. (44):

$$
z \frac{\partial^{2} U(z)}{\partial z^{2}}+\left(\frac{1}{2}-z\right) \frac{\partial U(z)}{\partial z}-\frac{\Omega^{2}}{4 c} U(z)=0 .
$$

Its solution could be expressed by Kummer functions, which is shown as

$$
U(z)=c_{1} \mathrm{KM}\left(\frac{\Omega^{2}}{4 c}, \frac{1}{2}, z\right)+c_{2} \mathrm{KU}\left(\frac{\Omega^{2}}{4 c}, \frac{1}{2}, z\right),
$$

where $c_{1}$ and $c_{2}$ are constants, $\operatorname{KM}(\alpha, \beta, z)$ and $\operatorname{KU}(\alpha, \beta, z)$ are two kinds of Kummer functions. Using $x=L \sqrt{-\frac{z}{c}}$, one can find $z=-c \xi^{2}$. Then substituting it into Eq. (45), the mode function reads

$$
U(\xi)=c_{1} \mathrm{KM}\left(\frac{\Omega^{2}}{4 c}, \frac{1}{2},-c \xi^{2}\right)+c_{2} \mathrm{KU}\left(\frac{\Omega^{2}}{4 c}, \frac{1}{2},-c \xi^{2}\right) .
$$

Substitution of the boundary condition with two fixed ends Eq. (27a) into Eq. (45) yields Eq. (47):

$$
\left(\begin{array}{l}
\mathrm{KM}\left(\frac{\Omega^{2}}{4 c}, \frac{1}{2}, 0\right) \quad \mathrm{KU}\left(\frac{\Omega^{2}}{4 c}, \frac{1}{2}, 0\right) \\
\mathrm{KM}\left(\frac{\Omega^{2}}{4 c}, \frac{1}{2},-c\right) \\
\mathrm{KU}\left(\frac{\Omega^{2}}{4 c}, \frac{1}{2},-c\right)
\end{array}\right)\left(\begin{array}{l}
c_{1} \\
c_{2}
\end{array}\right)=0 .
$$

one could find its mode frequency equation Eq. (48):

$\mathrm{KM}\left(\frac{\Omega^{2}}{4 c}+\frac{1}{2}, \frac{3}{2},-c\right)=0$.

In this section, we calculate the mode frequency of the non-uniform rod in previous subsection with three methods, i.e. the proposed method in Section 4, the WKB method in Section 5 and the method using Kummer function in this section. For the boundary condition with two fixed ends, the proposed method in Section 4 leads to the frequency equation shown as Eq. (28). For the rod with variable cross- section area $A(x)=A_{0} \exp \left(\frac{c x^{2}}{L^{2}}\right)$, i.e. $A(\xi)=A_{0} \exp \left(c \xi^{2}\right)$, the fifth order mode approximate frequency equation of the proposed method is shown as

$$
n \pi=\int_{0}^{1} \sqrt{\Omega_{j}^{2}-c-c^{2} \xi^{2}+\frac{c^{2} / 2}{\Omega_{j}^{2}-c-c^{2} \xi^{2}}+\frac{5 c^{4} \xi^{2}}{4\left(\Omega_{j}^{2}-c-c^{2} \xi^{2}\right)^{2}}+\frac{-9 c^{4} / 8}{\left(\Omega_{j}^{2}-c-c^{2} \xi^{2}\right)^{3}}+\frac{-27 c^{6} \xi^{2} / 2}{\left(\Omega_{j}^{2}-c-c^{2} \xi^{2}\right)^{4}}} d \xi, n=1,2,3, \ldots .
$$

As for the WKB method, the fifth order approximate mode frequency equation is shown as $n \pi=\Omega-\frac{c(3+c)}{6 \Omega}-\frac{c^{2}\left(3 c^{2}+10 c-15\right)}{120 \Omega^{3}}-\frac{c^{3}\left(-525+15 c^{3}-455 c+63 c^{2}\right)}{1680 \Omega^{5}}-\frac{c^{4}\left(-6300 c-2898 c^{2}+1323+35 c^{4}+180 c^{3}\right)}{8064 \Omega^{7}}, n=1,2,3, \ldots($

Table 1 Mode frequencies with two fixed ends and $c=0.5$

\begin{tabular}{ccllll}
\hline Mode Number & Kummer function & Methods & First order & Third order & Fifth order \\
\hline \multirow{2}{*}{1} & \multirow{2}{*}{3.231130281} & The proposed. & 3.233122144 & 3.231117984 & 3.231130556 \\
& \multirow{2}{*}{2} & WKB & 3.141592654 & 3.231284844 & 3.231128547 \\
& \multirow{2}{*}{6.329186675} & The proposed. & 6.329438059 & 6.329186583 & 6.329186675 \\
3 & \multirow{2}{*}{9.455600357} & WKB & 6.283185308 & 6.329192064 & 6.329186672 \\
& \multirow{2}{*}{12.58952819} & The proposed. & 9.455674941 & 9.455600352 & 9.455600357 \\
4 & & ThB proposed. & 12.58955966 & 12.58952819 & 12.58952819 \\
& & WKB & 12.56637062 & 12.58952836 & 12.58952819 \\
\hline
\end{tabular}


The values in Tables 1 are calculated by Eqs. (48), (49) and (50). In this case, first order, third order and fifth order means the values in corresponding column are calculated by the first order, third order and fifth order approximation mode frequency equations. The values calculated by Kummer functions are the exact values. The three tables indicate that the convergent speed of the proposed method is faster than that of the WKB method, which is in a good agreement with the theoretical analysis in Section 5. Particularly, the first order approximation of the proposed method has a satisfactory accuracy.

\section{Conclusions}

The proposed method results in a series solution of the free axial vibration of non-uniform rods. The problem also could be investigated by the WKB method, which also leads to a series solution.

The series solution of the WKB method could be seen as a Taylor expansion of the solution of the proposed method at infinity. In a special case, the series solution resulting from the proposed method is reduced to an exact solution with a concise form. However, the WKB method leads to a series with infinite terms.

The comparison indicates that the WKB method is simpler. However, its convergent speed is slower than that of the proposed method, which is also validated numerically using an exact solution of a kind of non-uniform rod with Kummer functions. Particularly, the first order approximation of the proposed method has a satisfactory accuracy.

\section{Acknowledgement}

This research was supported by the National Natural Science Fundation of China (Grant Nos. 11072157 and 10932006) and the Program for Changjiang Scholars and Innovative Research Team in University (IRT0971).

\section{Reference}

[1] M. Eisenberger, Exact axial vibration frequencies of a variable cross-section rod, Appl Acoust. 34(1991) 123-130.

[2] K. F. Graf, Wave Motion in Elastic Solids, Coulumbus, Ohio State University Press, 1975.

[3] S. Abrate, Vibration of non-uniform rods and beams, J Sound Vib. 185(1995) 703-716.

[4] C. N. Bapat, Vibration of rods with uniformly tapered sections, J Sound Vib. 185(1995) 185-189.

[5] B. M. Kumar, R. I. Sujth, Exact solutions for the axial vibration of non-uniformed rods, J Sound Vib. 207(1997) 721-729.

[6] R. Anil, R. I.Sujith, Closed-form solutions for the free axial vibration of inhomogeneous rods, J Sound Vib. 283(2005) 1015-1030

[7] S. Q. Guo, Z. Zhang, S. P. Yang, Free axial vibrations of a non-uniform rod, J Shijiazhuang Tiedao U (Natural Science). 23(2010): 59-63 (In Chinese).

[8] S. Q. Guo, S. P. Yang, Axial waves in one dimensional non-uniform waveguides, Theor Appl Mech Lett. 1( 2011) 021007

[9] W. F. Zhong, G. H. Nie, Scattering theory of elastic waves. Wuhan: Huangzhong University of Science \& Technology Press, 1997. 379-380. (in Chinese).

[10] R. E. Langer, The asymptotic solutions of ordinary linear differential equations of the second order, with special reference to the Stokes phenomenon. B Am Math Soc. 40(1934) 545-582. 